\title{
Molecular Characteristics of ST1193 Clone among Phylogenetic Group B2 Non-ST131 Fluoroquinolone-Resistant Escherichia coli
}

\author{
Jing Wu ${ }^{1+}$, Fangjun Lan ${ }^{2+}$, Yanfang $\mathrm{Lu}^{2,3}$, Qingwen $\mathrm{He}^{2}$ and Bin $\mathrm{Li}^{2 *}$
}

'Department of Rheumatology and Clinical Immunology, Zhujiang Hospital of Southern Medical University, Guangzhou, China, ${ }^{2}$ Department of Clinical Laboratory, Fujian Medical University Union Hospital, Fuzhou, China, ${ }^{3}$ Department of Clinical Laboratory, Maternal and Children's Health Hospital of Fujian Province, Fuzhou, China

OPEN ACCESS

Edited by:

Miklos Fuzi,

Semmelweis University, Hungary

Reviewed by:

Ghassan M. Matar

American University of Beirut,

Lebanon

Jorge Blanco,

Universidade de Santiago

de Compostela, Spain

*Correspondence:

Bin Li

leonlee307@hotmail.com

tThese authors have contributed equally to this work.

Specialty section:

This article was submitted to

Antimicrobials, Resistance

and Chemotherapy,

a section of the journal

Frontiers in Microbiology

Received: 29 July 2017 Accepted: 07 November 2017 Published: 21 November 2017

Citation:

Wu J, Lan F, Lu Y, He Q and Li B

(2017) Molecular Characteristics of ST1193 Clone among Phylogenetic Group B2 Non-ST131

Fluoroquinolone-Resistant Escherichia coli. Front. Microbiol. 8:2294. doi: 10.3389/fmicb.2017.02294
Objectives: Sequence type 1193 is emerging as a new, virulent and resistant lineage among fluoroquinolone resistant Escherichia coli (FQ⿱ $E$. coli). In this study, we investigated the prevalence and molecular characteristics of this clone isolated from a Chinese university hospital.

Methods: 73 phylogenetic group B2-FQ ${ }^{r}$-non-ST131 isolates were collected from August 2014 and August 2015 at a Chinese university hospital. Isolates were screened for ST1193 by multilocus sequence typing. E. coli ST1193 then underwent lactose fermentation determination, susceptibility testing, virulence genotyping, PCR-based $O$ typing, pulsed-field gel electrophoresis (PFGE) and FQ ${ }^{r}$ mechanism analysis.

Results: Of 73 B2-FQ ${ }^{r}$-non ST131 E. coli isolates, 69.9\% $(n=51)$ were ST1193. 90.2\% (46/51) of ST1193 isolates were O75 serotype and $96.1 \%$ (49/51) of the ST1193 isolates were lactose non-fermenters. 35 clusters were identified by PFGE. ST1193 isolates exhibited a set of 3 conserved mutations defining quinolone-resistance determining region substitutions (gyrA S83L, D87N, and parC S80I). The most frequent VF genes detected in these E. coli ST1193 isolates were fyuA (yersiniabactin, 96.1\%), fimH (type 1 fimbriae, 94.1\%), iutA (iron uptake gene, 90.2\%), kpsMT II (group II capsule, 90.2\%), kpsK1 (K1 capsule, 86.3\%) and PAl.

Conclusion: ST1193 lineage accounts for the majority of group B2-FQ'-nonST131 E. coli clinical isolates. Most of the ST1193 are serotype O75 and lactose non-fermenting. Strategic surveillance and control schemes are needed in the future for this newly emerging clone of E. coli: B2-FQ'-ST1193.

Keywords: Escherichia coli, ST1193, fluoroquinolone resistance, phylogenetic group B2, 075

\section{INTRODUCTION}

Escherichia coli (E. coli) is commonly found in the gut flora of humans and can cause many different kinds of extra-intestinal infections, such as septicemias, meningitis and urinary tract infections (Pitout, 2012; Poolman and Wacker, 2016). Many methods can be used for typing E. coli, including serotyping, pulsed-field gel electrophoresis (PFGE) and various PCR-based molecular methods 
(Jonas et al., 2003; Cespedes et al., 2017; Roer et al., 2017). Multilocus sequence typing (MLST) is a PCR-based molecular typing method and is considered the "gold standard" subtyping technique that can usually be used to detect the genetic relatedness between strains and identify a specific clone with high discriminatory ability for various bacterial pathogens (Maiden et al., 2013; Perez-Losada et al., 2013). The method is now widely used in the identification and classification of E. coli. Successful MLST clones of E. coli have been reported in various regions of the world (Nicolas-Chanoine et al., 2014; Dautzenberg et al., 2016). E. coli ST131 is a classic clonal group from phylogenetic group B2. It typically possesses a wide variety of virulence factors (VFs) and is strongly associated with resistance to fluoroquinolone and extended spectrum cephalosporins (Nicolas-Chanoine et al., 2014; Dautzenberg et al., 2016).

Simultaneously, ST1193 has been reported recently, as a new, virulent and resistant E. coli that commonly causes extraintestinal infections (Platell et al., 2012; Chang et al., 2014; Matsumura et al., 2017). E. coli ST1193 shares many common features, including fluoroquinolone resistance $\left(\mathrm{FQ}^{\mathrm{r}}\right)$, lactose non-fermenting and phylogenetic group B2 (Platell et al., 2012; Chang et al., 2014). In China, E. coli ST1193 has been sporadically reported (Zhao et al., 2015; Xia et al., 2017). However, no data was available as to the detailed characteristics of the lineage in this region. The aim of this study was to detect the prevalence and molecular characteristics of ST1193 among phylogenetic group B2-FQ ${ }^{\mathrm{r}}$-non-ST131 E. coli in China.

\section{MATERIALS AND METHODS}

\section{Bacterial Isolates}

A total of 700 non-duplicate E. coli clinical isolates were consecutively collected at a Chinese university hospital (Fujian Medical University Union Hospital, Fuzhou, China) between August 2014 and August 2015. 250 phylogenetic group B2 isolates were identified using PCR method as previously described (Clermont et al., 2013). Group B2 isolates were then tested for susceptibility to ciprofloxacin (CIP) by disk diffusion test according to CLSI (CLSI, 2017). B2-FQ ${ }^{r}$ E. coli isolates then underwent the PCR-based method for ST131-associated SNPs in $m d h$ and $g y r B$ as previously described (Johnson et al., 2009).

\section{MLST and Further Isolate Selection}

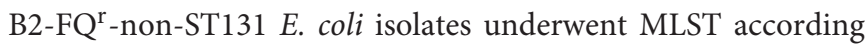
to the Achtman scheme using seven housekeeping genes (adk, fum $C, \operatorname{gyr} B, i c d, m d h$, purA, and $\operatorname{rec} A)^{1}$. E. coli ST1193 isolates were further characterized in detail below in this study.

\section{Susceptibility Testing and Lactose Fermentation Determination}

Antimicrobial susceptibility of E. coli ST1193 isolates was determined by disk diffusion test to nine antimicrobials, which are commonly used in clinical therapy. The antibiotics tested

${ }^{1}$ http://mlst.ucc.ie/mlst/dbs/Ecoli in this study were as follows: aztreonam (30 $\mu \mathrm{g})$, cefotaxime (30 $\mu \mathrm{g})$, ceftazidime (30 $\mu \mathrm{g})$, cefepime (30 $\mu \mathrm{g})$, ertapenem $(10 \mu \mathrm{g})$, imipenem $(10 \mu \mathrm{g})$, piperacillin-tazobactam $(100 / 10 \mu \mathrm{g})$, amikacin (30 $\mu \mathrm{g})$, and trimethoprim-sulfamethoxazole $(1.25 / 23.75 \mu \mathrm{g})$. All disks were obtained from Oxide, Ltd (Cambridge, United Kingdom). The results were interpreted according to the breakpoints of the CLSI criteria (CLSI, 2017). E. coli ATCC 25922 was used for routine quality control. Lactose fermentation was determined using KIA (Kligler's Iron Agar).

\section{O Typing}

Molecular serotyping of E. coli ST1193 isolates was performed by a multiplex PCR method to detect 16 serogroups, including $\mathrm{O} 1$, O2, O4, O6, O7, O8, O12, O15, O16, O18, O21, O22, O25, O75, O83, and O157 (DebRoy et al., 2011). Isolates that could not be typed using this method were classified as non-typeable in this study.

\section{Pulsed-Field Gel Electrophoresis}

In order to determine the genetic relateness of the E. coli ST1193 isolates, isolates were subjected to PFGE analysis using XbaI digestion as previously described (Li et al., 2011). A PFGE dendrogram was constructed with BioNumerics software (Applied Maths, Sint-Martens-Latem, Belgium) according to the unweighted pair group method based on Dice coefficients. Isolates with a Dice similarity index $\geq 85 \%$ were considered to belong to the same PFGE group (Gibreel et al., 2012; Hussain et al., 2012).

\section{Virulence Gene Profiling}

The presence of 26 known VFs genes was screened by a multiplex PCR method (Johnson and Stell, 2000). For each isolate, the virulence score (VF score) was calculated as the sum of all virulence-associated genes detected in this study. The sum of all the VF scores of the isolates was then calculated, and finally this sum was divided by the number of isolates to give the mean VF score.

\section{Detection of Fluoroquinolone Resistance Genes and blacTX-M Gene}

Escherichia coli ST1193 isolates resistant to cefotaxime and/or ceftazidime underwent detection of bla $a_{\text {CTX-M using PCR and }}$ sequencing (Li et al., 2011). As to all ST1193 isolates, mutations in the quinolone-resistance determining region (QRDR) in chromosomal gyrA, parC and parE genes were determined by PCR and sequencing (Everett et al., 1996). The presence of plasmid mediated quinolone resistance determinants [PMQRs; $q n r A, q n r B, q n r C, q n r D$, qnrS, and $\left.a a c\left(6^{\prime}\right)-I b-c r\right]$ were detected by PCR as previously described (Chen et al., 2012).

\section{RESULTS}

\section{Bacterial Isolates}

One hundred and thirty one $\mathrm{B} 2-\mathrm{FQ}^{\mathrm{r}}$ E. coli isolates were collected in this study, including 58 ST131 and 73 non-ST131 
strains. $73 \mathrm{~B} 2-\mathrm{FQ}^{\mathrm{r}}$-non ST131 E. coli isolates were grouped into 10 distinct STs, with the predominant ST being ST1193 ( $n=51,69.9 \%)$, followed by ST95 $(n=4,5.5 \%)$, ST140 $(n=3,4.1 \%)$, ST648 $(n=3,4.1 \%)$, ST73 $(n=3,4.1 \%)$, ST92 $(n=2,2.7 \%)$, ST117 $(n=2,2.7 \%)$, ST354 $(n=2,2.7 \%)$, ST1318 $(n=1,1.4 \%)$, ST2558 $(n=1,1.4 \%)$, and ST3177 $(n=1,1.4 \%)$. The majority of $E$. coli ST1193 strains (34, $66.7 \%)$ were isolated from urine, followed by blood $(n=7$, $13.7 \%)$, secretions $(n=6,11.8 \%)$, fluids $(n=3,5.9 \%)$, and pus $(n=1,2.0 \%)$. Females accounted for $72.5 \%(37 / 51)$ of the ST1193 isolates, and the average age was 65 years (range: 1091 years). E. coli ST1193 strains were isolated from different wards (Supplementary Table S1).

\section{O Typing and Lactose Fermentation Determination}

90.2\% (46/51) of the E. coli ST1193 were classified as O-type O75. The other five isolates could not be typed. $96.1 \%(49 / 51)$ of the ST1193 isolates were lactose non-fermenters, including 46 O75 and 3 non-O typeable isolates.

\section{Antimicrobial Susceptibility Testing}

Escherichia coli ST1193 isolates showed high resistance against trimethoprim-sulfamethoxazole $(64.7 \%)$. Resistance rates were low than $50 \%$ to cefotaxime $(45.1 \%)$, aztreonam $(21.6 \%)$, cefepime (19.6\%), ceftazidime $(9.8 \%)$, and amikacin $(7.8 \%)$, respectively. All the strains were susceptible to imipenem and ertapenem.

\section{Bacterial Clonal Relatedness}

Pulsed-field gel electrophoresis patterns of the 51 E. coli ST1193 isolates identified 35 PFGE types (clusters 1-35) (Figure 1). No single dominant intra-hospital PFGE type was detected when using a cutoff of $85 \%$ similarity. Three PFGE subtypes (clusters 1,2 , and 3) encompassed at least three ST1193 isolates.

\section{Fluoroquinolone Resistance Characteristics and bla}

All of the 51 E. coli ST1193 isolates contained a set of 3 conserved mutations defining QRDR substitutions (gyrA S83L, D87N, and parC S80I). Two ST1193 isolates had additional mutations in the QRDR of parC (N167Y). No mutation was found in parE. Meanwhile, two isolates harbored $a a c\left(6^{\prime}\right)-I b-c r$ variant and none of other PMQR determinants ( $q n r A, \mathrm{q} n r B, q n r C, q n r D, q n r S$, oqxAB, and qepA) were detected in this study.

Among 23 cefotaxime and/or ceftazidime E. coli ST1193 isolates, 22 (95.7\%) contained bla $a_{\mathrm{CTX}-\mathrm{M}}$ genes. Of these $b l a_{\mathrm{CTX}-\mathrm{M}}$ positive isolates, 12 isolates carried $b l a_{\mathrm{CTX}-\mathrm{M}-14}, 6$ isolates carried bla $a_{\mathrm{CTX}-\mathrm{M}-15}$ and 2 isolates carried bla $a_{\mathrm{CTX}-\mathrm{M}-123}$. The remaining 2 isolates co-harbored bla $a_{\mathrm{CTX}-\mathrm{M}-14}$ and $b l a_{\mathrm{CTX}-\mathrm{M}-15}$. bla $a_{\mathrm{CTX}-\mathrm{M}-2 \text {, }}$ bla $a_{\mathrm{CTX}-\mathrm{M}-8}$ and bla $a_{\mathrm{CTX}-\mathrm{M}-25}$ groups were not detected in these isolates.

\section{Virulence Gene Profiling}

The most frequent VF genes were fyuA (yersiniabactin, 96.1\%), fimH (type 1 fimbriae, 94.1\%), iutA (iron uptake gene, 90.2\%),
kpsMT II (group II capsule, 90.2\%), kpsK1 (K1 capsule, 86.3\%) and PAI $(86.3 \%)$, each of which was detected in $\geq 80 \%$ of the isolates. Four genes were identified in less than $10 \%$ of isolates, including kpsK5 (7.8\%), sfa/focDE (7.8\%), traT (5.9\%), and papG allele II (2.0\%). kpsMTIII, papAH, papEF, papC, papG alleleI, $n f a E, r f c, p a p G I I / I I I, h l y A, s f a S, g a f D, c v a C, c d t B, f o c G, c n f 1$, and $a f a / d r a B C$ were not detected in this study.

\section{DISCUSSION}

Fluoroquinolones are the most widely used antimicrobial agents for the treatment of different infections in China. The high frequency of fluoroquinolone resistance in E. coli is considered as a feature of clinical bacteriology in the last 10 years in China according to a CHINET report (Hu et al., 2016). Fluoroquinolone resistance usually occurred in low-virulence E. coli phylogenetic group $\mathrm{A}$ isolates rather than in isolates from groups B2 and D. However, the recent worldwide emergence of $\mathrm{B} 2-\mathrm{FQ}^{\mathrm{r}}-E$. coli has occurred primarily through clonal expansion of E. coli ST131 (Nicolas-Chanoine et al., 2014; Dautzenberg et al., 2016). Our study confirmed that while ST131 is still the predominant subclone among B2-FQ ${ }^{\mathrm{r}}$-E. coli clinical isolates in China, ST1193 is emerging as an important subgroup and accounts for a similar percentage with ST131 among group B2-FQ ${ }^{r}-E$. coli. The reasons for the expansion of such subclone are unclear and it is worth investigating in future study to explain its dissemination in China.

Historically, E. coli serotype O75 isolates are strongly associated with urinary tract infection and sepsis in humans, and virulence-associated phylogenetic group B2 (Devine et al., 1989; Obata-Yasuoka et al., 2002; Platell et al., 2012). Moreover, O75 strains are usually non-lactose fermenter (Platell et al., 2012; Chang et al., 2014). Our results were consistent with the results from previous studies as the majority of our ST1193 isolates were non-lactose fermenter (96.1\%) and belonged to serotype O75 (90.2\%). Because O75 isolates were never found to be associated with FQ resistance in previous studies, the recent emergence of O75-B2-FQ ${ }^{r}-\mathrm{ST} 1193$ E. coli isolates are probably due to clonal expansion (Platell et al., 2012).

In this study, ciprofloxacin resistance was mainly due to the accumulation of three point mutations in DNA gyrase or topoisomerase IV (gyrA S83L, D87N, and parC S80I), which are the primary bacterial target of quinolone (Hooper and Jacoby, 2015; Correia et al., 2017). The pattern of non-synonymous mutations found in our B2-FQ ${ }^{\mathrm{r}}-\mathrm{ST} 1193$ isolates was identical to those observed in Australian and Korean ST1193 isolates (Platell et al., 2012; Chang et al., 2014). As discussed in previous study (Platell et al., 2012), the homogeneity of ST1193 isolates suggests this subclone emerged and spreaded from a common ancestor. Meanwhile, previous study suggested that more distinctive QRDR mutations probably provided E. coli ST131 $\mathrm{H} 30$ isolates with fitness advantages over other fluoroquinolone resistant E. coli isolates (Johnson et al., 2015). In this study, all the E. coli ST1193 isolates contained the same distinctive combination of three non-synonymous mutations (gyrA S83L, D87N, and parC S80I). These three QRDR mutations found in 


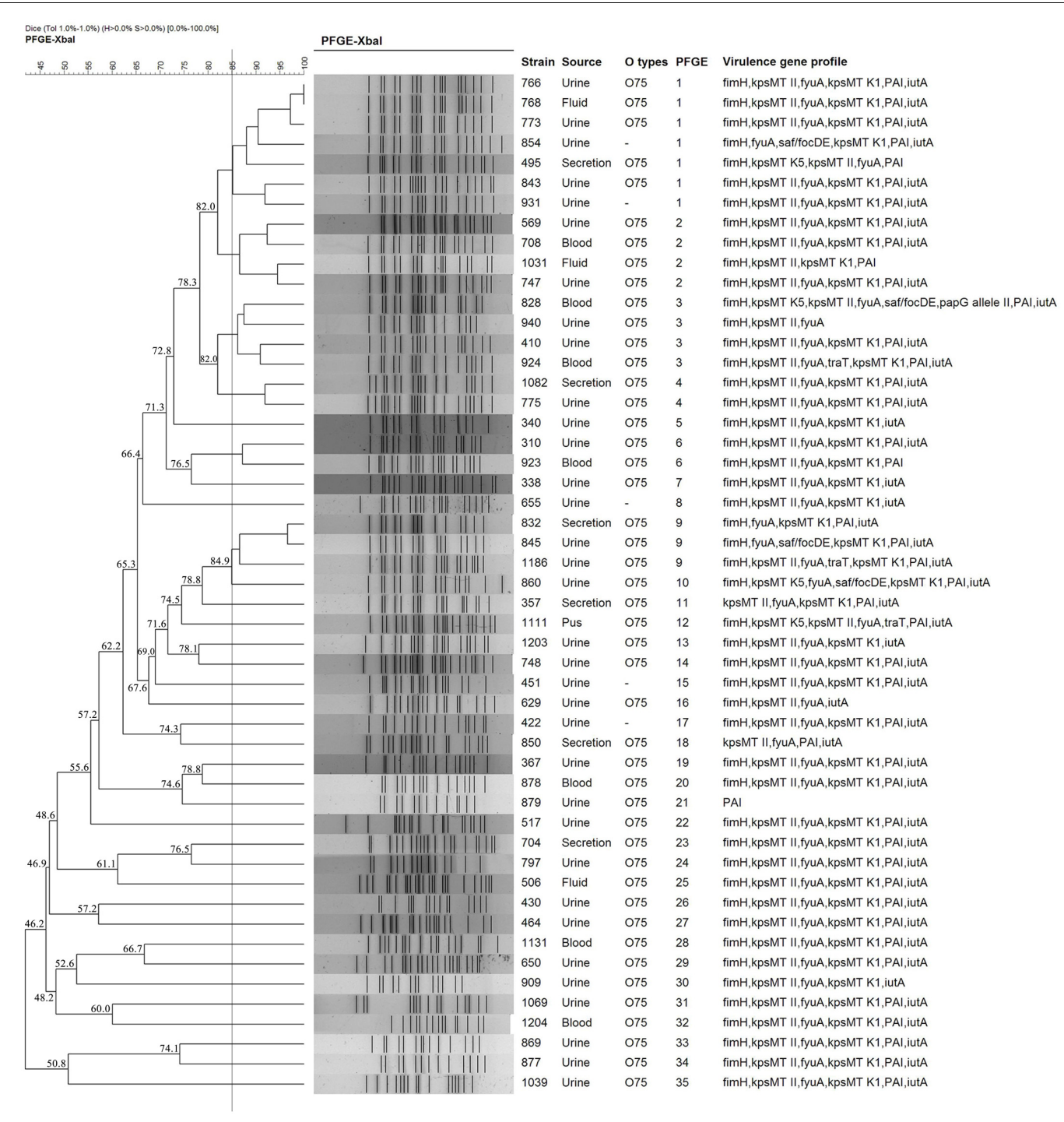

FIGURE 1 | Dendrogram of the PFGE patterns of chromosomal DNA restriction fragments from 51 ST1193 Escherichia coli isolates. The dendrogram was constructed using the UPGMA method. Dice coefficients (percentages) are listed in the scale on the top of the dendrogram. Strain number, sample source, $O$ serotype, PFGE patterns and virulence factors (VFs) are included.

ST1193 isolates are the only ones that have been experimentally proved to increase fluoroquinolone MICs at the present time (Marcusson et al., 2009). Therefore, non-synonymous QRDR mutations in gyrA and parC may play a similar role in the fitness of E. coli ST1193 isolates as what they do in ST131 lineage.

The clonal dissemination of blaCTX-M-55 among E. coli isolates causing urinary tract infections maybe due to the expansion of ST1193 clone in one previous study (Xia et al., 2017). In this study, we attempted to identify the blaCTX-M genes in our third generation cephalosporin resistant ST1193 isolates. Although our results showed a high percentage (95.7\%) of third generation cephalosporin resistant isolates harboring different blaCTX-M genes, no correlation between ST1193 clone and any special blaCTX-M genes was found in this study.
Regional difference may be the main reason for the disparity of these two studies. Further nationwide study should be carried out to explain the difference in China.

Identical virulence gene profiles as one common phenotypic characteristic were presenting in previous studies (Platell et al., 2012; Cremet et al., 2013). In this study, we also exhibited identical virulence genes profile (fimH, fyuA, iutA, $k p s M T$ II, kpsKl, and PAI) among most of the ST1193 isolates. Together with the results of serotyping, fluoroquinolone resistance and phylogenetic group B2, the high level of homogeneity between ST1193 strains from different geographical regions suggests a probable divergence from a common ancestor.

Meanwhile, we identified a total of 35 PFGE types using PFGE analysis in this study, and no dominant intra-hospital PFGE type 
was found using a cutoff of $85 \%$ similarity. Our results suggested that E. coli ST1193 clinical isolates were not spread by clonal strains in our hospital.

\section{CONCLUSION}

ST1193 lineage account for the majority of B2-FQ ${ }^{r}$-non-ST131 E. coli population in this study. These ST1193 isolates are highly clonal diversity as indicated by PFGE patterns. Most of the ST1193 isolates were serotype O75 and lactose non-fermenting. Strategic surveillance and control schemes are needed in the future for this newly emerging clone of E. coli: B2-FQ ${ }^{\mathrm{r}}$-ST1193.

\section{ETHICS STATEMENT}

This study was approved by The Institutional Review Board of the Fujian Medical University Union Hospital, Fuzhou, China. No consent was needed for this study.

\section{AUTHOR CONTRIBUTIONS}

JW, FL, YL, and QH performed experiments. JW and FL conceived the study and analyzed the results. BL supervised

\section{REFERENCES}

Cespedes, S., Saitz, W., Del Canto, F., De la Fuente, M., Quera, R., Hermoso, M., et al. (2017). Genetic diversity and virulence determinants of Escherichia coli strains isolated from patients with Crohn's disease in Spain and Chile. Front. Microbiol. 8:639. doi: 10.3389/fmicb.2017.00639

Chang, J., Yu, J., Lee, H., Ryu, H., Park, K., and Park, Y. J. (2014). Prevalence and characteristics of lactose non-fermenting Escherichia coli in urinary isolates. J. Infect. Chemother. 20, 738-740. doi: 10.1016/j.jiac.2014.07.005

Chen, X., Zhang, W., Pan, W., Yin, J., Pan, Z., Gao, S., et al. (2012). Prevalence of qnr, aac(6')-Ib-cr, qepA, and oqxAB in Escherichia coli isolates from humans, animals, and the environment. Antimicrob. Agents Chemother. 56, 3423-3427. doi: 10.1128/AAC.06191-11

Clermont, O., Christenson, J. K., Denamur, E., and Gordon, D. M. (2013). The Clermont Escherichia coli phylo-typing method revisited: improvement of specificity and detection of new phylo-groups. Environ. Microbiol. Rep. 5, 58-65. doi: 10.1111/1758-2229.12019

CLSI (2017). M100S. Performance Standards for Antimicrobial Susceptibility Testing, 27th Edn. Wayne, PA: CLSI.

Correia, S., Poeta, P., Hebraud, M., Capelo, J. L., and Igrejas, G. (2017). Mechanisms of quinolone action and resistance: where do we stand? J. Med. Microbiol. 66, 551-559. doi: 10.1099/jmm.0.000475

Cremet, L., Caroff, N., Giraudeau, C., Reynaud, A., Caillon, J., and Corvec, S. (2013). Detection of clonally related Escherichia coli isolates producing different CMY beta-lactamases from a cystic fibrosis patient. J. Antimicrob. Chemother. 68, 1032-1035. doi: 10.1093/jac/dks520

Dautzenberg, M. J., Haverkate, M. R., Bonten, M. J., and Bootsma, M. C. (2016). Epidemic potential of Escherichia coli ST131 and Klebsiella pneumoniae ST258: a systematic review and meta-analysis. BMJ Open 6:e009971. doi: 10.1136/ bmjopen-2015-009971

DebRoy, C., Roberts, E., and Fratamico, P. M. (2011). Detection of O antigens in Escherichia coli. Anim. Health Res. Rev. 12, 169-185. doi: 10.1017/ S1466252311000193 the study and prepared the manuscript. All authors read and approved the final manuscript.

\section{FUNDING}

This study was supported by the National Natural Science Foundation of China (grant no. 81601397), the Fujian Provincial Funds for Distinguished Young Scientists in Colleges and Universities, China (grant no. JA13134), and the Medical Elite Cultivation Program of Fujian, China (grant no. 2015-ZQN-ZD-15).

\section{ACKNOWLEDGMENT}

The authors thank Prof. Min Chen and Department of Laboratory Medicine, Medical Technology and Engineering College, Fujian Medical University for their kind help.

\section{SUPPLEMENTARY MATERIAL}

The Supplementary Material for this article can be found online at: https://www.frontiersin.org/articles/10.3389/fmicb. 2017.02294/full\#supplementary-material

Devine, D. A., Robinson, L., and Roberts, A. P. (1989). Occurrence of K1, K5 and O antigens in Escherichia coli isolates from patients with urinary tract infections or bacteraemia. J. Med. Microbiol. 30, 295-299. doi: 10.1099/00222615-30-4-295

Everett, M. J., Jin, Y. F., Ricci, V., and Piddock, L. J. (1996). Contributions of individual mechanisms to fluoroquinolone resistance in 36 Escherichia coli strains isolated from humans and animals. Antimicrob. Agents Chemother. 40, 2380-2386.

Gibreel, T. M., Dodgson, A. R., Cheesbrough, J., Fox, A. J., Bolton, F. J., and Upton, M. (2012). Population structure, virulence potential and antibiotic susceptibility of uropathogenic Escherichia coli from Northwest England. J. Antimicrob. Chemother. 67, 346-356. doi: 10.1093/jac/dkr451

Hooper, D. C., and Jacoby, G. A. (2015). Mechanisms of drug resistance: quinolone resistance. Ann. N. Y. Acad. Sci. 1354, 12-31. doi: 10.1111/nyas.12830

Hu, F. P., Guo, Y., Zhu, D. M., Wang, F., Jiang, X. F., Xu, Y. C., et al. (2016). Resistance trends among clinical isolates in China reported from CHINET surveillance of bacterial resistance, 2005-2014. Clin. Microbiol. Infect. 22(Suppl. 1), S9-S14. doi: 10.1016/j.cmi.2016.01.001

Hussain, A., Ewers, C., Nandanwar, N., Guenther, S., Jadhav, S., Wieler, L. H., et al. (2012). Multiresistant uropathogenic Escherichia coli from a region in India where urinary tract infections are endemic: genotypic and phenotypic characteristics of sequence type 131 isolates of the CTX-M-15 extendedspectrum-beta-lactamase-producing lineage. Antimicrob. Agents Chemother. 56, 6358-6365. doi: 10.1128/AAC.01099-12

Johnson, J. R., Johnston, B., Kuskowski, M. A., Sokurenko, E. V., and Tchesnokova, V. (2015). Intensity and mechanisms of fluoroquinolone resistance within the $\mathrm{H} 30$ and H30Rx subclones of Escherichia coli sequence type 131 compared with other fluoroquinolone-resistant E. coli. Antimicrob. Agents Chemother. 59, 4471-4480. doi: 10.1128/AAC.00673-15

Johnson, J. R., Menard, M., Johnston, B., Kuskowski, M. A., Nichol, K., and Zhanel, G. G. (2009). Epidemic clonal groups of Escherichia coli as a cause of antimicrobial-resistant urinary tract infections in Canada, 2002 to 2004. Antimicrob. Agents Chemother. 53, 2733-2739. doi: 10.1128/AAC. 00297-09 
Johnson, J. R., and Stell, A. L. (2000). Extended virulence genotypes of Escherichia coli strains from patients with urosepsis in relation to phylogeny and host compromise. J. Infect. Dis. 181, 261-272. doi: 10.1086/315217

Jonas, D., Spitzmuller, B., Weist, K., Ruden, H., and Daschner, F. D. (2003). Comparison of PCR-based methods for typing Escherichia coli. Clin. Microbiol. Infect. 9, 823-831. doi: 10.1046/j.1469-0691.2003.00661.x

Li, B., Sun, J. Y., Liu, Q. Z., Han, L. Z., Huang, X. H., and Ni, Y. X. (2011). High prevalence of CTX-M beta-lactamases in faecal Escherichia coli strains from healthy humans in Fuzhou, China. Scand. J. Infect. Dis. 43, 170-174. doi: 10.3109/00365548.2010.538856

Maiden, M. C., Jansen van Rensburg, M. J., Bray, J. E., Earle, S. G., Ford, S. A., Jolley, K. A., et al. (2013). MLST revisited: the gene-by-gene approach to bacterial genomics. Nat. Rev. Microbiol. 11, 728-736. doi: 10.1038/nrmicro3093

Marcusson, L. L., Frimodt-Moller, N., and Hughes, D. (2009). Interplay in the selection of fluoroquinolone resistance and bacterial fitness. PLOS Pathog. 5:e1000541. doi: 10.1371/journal.ppat.1000541

Matsumura, Y., Noguchi, T., Tanaka, M., Kanahashi, T., Yamamoto, M., Nagao, M., et al. (2017). Population structure of Japanese extraintestinal pathogenic Escherichia coli and its relationship with antimicrobial resistance. J. Antimicrob. Chemother. 72, 1040-1049. doi: 10.1093/jac/dkw530

Nicolas-Chanoine, M. H., Bertrand, X., and Madec, J. Y. (2014). Escherichia coli ST131, an intriguing clonal group. Clin. Microbiol. Rev. 27, 543-574. doi: 10.1128/CMR.00125-13

Obata-Yasuoka, M., Ba-Thein, W., Tsukamoto, T., Yoshikawa, H., and Hayashi, H. (2002). Vaginal Escherichia coli share common virulence factor profiles, serotypes and phylogeny with other extraintestinal E. coli. Microbiology 148, 2745-2752. doi: 10.1099/00221287-148-9-2745

Perez-Losada, M., Cabezas, P., Castro-Nallar, E., and Crandall, K. A. (2013). Pathogen typing in the genomics era: MLST and the future of molecular epidemiology. Infect. Genet. Evol. 16, 38-53. doi: 10.1016/j.meegid.2013.01.009

Pitout, J. D. (2012). Extraintestinal pathogenic Escherichia coli: a combination of virulence with antibiotic resistance. Front. Microbiol. 3:9. doi: 10.3389/fmicb. 2012.00009
Platell, J. L., Trott, D. J., Johnson, J. R., Heisig, P., Heisig, A., Clabots, C. R., et al. (2012). Prominence of an O75 clonal group (clonal complex 14) among non-ST131 fluoroquinolone-resistant Escherichia coli causing extraintestinal infections in humans and dogs in Australia. Antimicrob. Agents Chemother. 56, 3898-3904. doi: 10.1128/AAC.06120-11

Poolman, J. T., and Wacker, M. (2016). Extraintestinal pathogenic Escherichia coli, a common human pathogen: challenges for vaccine development and progress in the field. J. Infect. Dis. 213, 6-13. doi: 10.1093/infdis/ jiv429

Roer, L., Hansen, F., Thomsen, M. C., Knudsen, J. D., Hansen, D. S., Wang, M., et al. (2017). WGS-based surveillance of third-generation cephalosporin-resistant Escherichia coli from bloodstream infections in Denmark. J. Antimicrob. Chemother. 72, 1922-1929. doi: 10.1093/jac/dkx092

Xia, L., Liu, Y., Xia, S., Kudinha, T., Xiao, S. N., Zhong, N. S., et al. (2017). Prevalence of ST1193 clone and IncI1/ST16 plasmid in E-coli isolates carrying blaCTX-M-55 gene from urinary tract infections patients in China. Sci. Rep. 7:44866. doi: 10.1038/srep44866

Zhao, L., Zhang, J., Zheng, B., Wei, Z., Shen, P., Li, S., et al. (2015). Molecular epidemiology and genetic diversity of fluoroquinolone-resistant Escherichia coli isolates from patients with community-onset infections in 30 Chinese county hospitals. J. Clin. Microbiol. 53, 766-770. doi: 10.1128/JCM. 02594-14

Conflict of Interest Statement: The authors declare that the research was conducted in the absence of any commercial or financial relationships that could be construed as a potential conflict of interest.

Copyright (C) $2017 \mathrm{Wu}, \mathrm{Lan}, \mathrm{Lu}, \mathrm{He}$ and Li. This is an open-access article distributed under the terms of the Creative Commons Attribution License (CC BY). The use, distribution or reproduction in other forums is permitted, provided the original author(s) or licensor are credited and that the original publication in this journal is cited, in accordance with accepted academic practice. No use, distribution or reproduction is permitted which does not comply with these terms. 